\title{
Impurity and Trace Tritium Transport in Tokamak Edge Turbulence
}

\author{
V. Naulin \\ Association EURATOM-Risф National Laboratory, \\ OPL-128 Ris $\phi$, DK-4000 Roskilde, Denmark
}

(Dated: April 11, 2018)

\begin{abstract}
The turbulent transport of impurity or minority species, as for example Tritium, is investigated in driftAlfvén edge turbulence. The full effects of perpendicular and parallel convection are kept for the impurity species. The impurity density develops a granular structure with steep gradients and locally exceeds its initial values due to the compressibility of the flow. An approximate decomposition of the impurity flux into a diffusive part and an effective convective part (characterized by a pinch velocity) is performed and a net inward pinch effect is recovered. The pinch velocity is explained in terms of Turbulent Equipartition [1] and is found to vary poloidally. The results show that impurity transport modeling needs to be two-dimensional, considering besides the radial direction also the strong poloidal variation in the transport coefficients.
\end{abstract}

PACS numbers: $52.25 . \mathrm{Gj}, 52.35 . \mathrm{Ra}, 52.65 . \mathrm{Kj}$ 
The transport properties of impurities are of great concern in magnetic fusion devices. In particular impurities accumulate in the center of the plasma, where they are responsible for significant radiation losses. It is well established that turbulence is the key drive for plasma transport in the edge region and thus turbulence will also dominate the transport of impurities in that region. While in neutral fluids the transport of passive scalars is a very active field of research [2], it has in the context of plasma turbulence not yet found corresponding attention and measurements of impurity transport are most often interpreted within reduced 1D transport models [3, 4]. In plasma turbulence the velocity field is in general compressible what makes the transport and mixing more complex than in incompressible flows [2]. This puts strong demands on the numerical modeling.

Pinching of impurities has been observed as well as fast inwards transport of trace Tritium in JET Tritium puffs [5]. Especially in the edge region an inward convective flux (pinch) of impurites is found, which is proportional to the measured diffusion [3]. In the core where anomalous, turbulent transport is less important than in the edge the observed inward pinch of impurities seems to be in good agreement with neoclassical predictions based on trapped particle orbits. The PfirschSchlüter impurity transport in the edge [6], leads to an inward impurity pinch due to ion-impurity collisions. However, there is no explanation for the high inward pinch velocities needed to describe impurity transport through in the turbulent edge region and it's scaling with the effective diffusion.

Here we investigate the transport of impurities as a passive tracer field in electromagnetic edge turbulence described by a standard model of drift-Alfvén turbulence [7, 8, 9]. Flux tube geometry is used, with local slab-like coordinates $(x, y, s)$ [10]. The following equations for the fluctuations in density $n$, potential $\phi$ with associated vorticity $\omega=\nabla_{\perp}^{2} \phi$, current $J$ and parallel ion velocity $u$ arise in the usual drift-scaling:

$$
\begin{gathered}
\frac{\partial \omega}{\partial t}+\{\phi, \omega\}=\mathcal{K}(n)+\nabla_{\|} J+\mu_{\omega} \nabla_{\perp}^{2} \omega \\
\frac{\partial n}{\partial t}+\left\{\phi, n_{E Q}+n\right\}=\mathcal{K}(n-\phi)+\nabla_{\|}(J-u)+\mu_{n} \nabla_{\perp}^{2} n \\
\frac{\partial}{\partial t}\left(\widehat{\beta} A_{\|}+\widehat{\mu} J\right)+\widehat{\mu}\{\phi, J\}=\nabla_{\|}\left(n_{E Q}+n-\phi\right)-C J \\
\widehat{\varepsilon}\left(\frac{\partial u}{\partial t}+\{\phi, u\}\right)=-\nabla_{\|}\left(n_{E Q}+n\right) .
\end{gathered}
$$

In addition to the cross-field advection, the parallel derivatives carry non-linearities entering through $A_{\|}$, while the operator $\mathcal{K}$ represents effects of normal and geodesic curvature due to magnetic field inhomogeneity with $\omega_{B}=2 \frac{L_{\perp}}{R}, \mathrm{R}$ being the tokamak major radius and $L_{\perp}$ the mean 
gradient length of the pressure profile,

$$
\nabla_{\|} n=\frac{\partial n}{\partial s}-\widehat{\beta}\left\{A_{\|}, n\right\}, \quad \mathcal{K}(n)=-\omega_{B}\left(\sin s \frac{\partial n}{\partial x}+\cos s \frac{\partial n}{\partial y}\right) .
$$

The parallel current $J$ is connected to the magnetic potential given by $J=-\nabla_{\perp}^{2} A_{\|}$. The parameters reflect the competition between parallel and perpendicular dynamics, represented in the scale ratio $\widehat{\varepsilon}=\left(q R / L_{\perp}\right)^{2}$ where $q$ is the safety factor. The electron parallel dynamics is controlled by the parameters

$$
\widehat{\beta}=\frac{2 \mu_{0} p_{e, 0}}{B^{2}} \widehat{\varepsilon}, \quad \widehat{\mu}=\frac{m_{e}}{M_{i}} \widehat{\varepsilon}, \quad C=0.51 \frac{L_{\perp}}{\tau_{e} c_{s}} \widehat{\mu}=v \widehat{\mu},
$$

with $\tau_{e}$ being the electron collision time and the factor 0.51 reflects the parallel resistivity [11]. $n_{E Q}$ is an equilibrium density associated with corresponding neoclassical fields and currents. In detail the curvature operator on the equilibrium density balances the neoclassical Pfirsch-Schlüter current $J_{P S}$

$$
\mathcal{K}\left(n_{E Q}\right)+\nabla_{\|} J_{P S}=0
$$

where the Pfirsch-Schlüter current is driven by the corresponding neoclassical potential

$$
\nabla_{\text {II }}\left(\phi_{P S}\right)=C J_{P S}
$$

To determine the evolution of the impurity density $n_{\text {imp }}$ we assume that the contribution of the impurities to the gross plasma density $n_{i, 0}$ is negligible, i. e. $n_{i}=n_{i, 0}+n_{i m p} \approx n_{i, 0}$. In experiments the assumption $n_{i, 0} \gg n_{i m p}$ is possibly not always fulfilled, especially not during the initial phase of a Tritium gas puff, where locally in the edge region the Tritium migth contribute a significant part of overall plasma density. The bulk plasma is quasineutral with $n_{e}=n_{i, 0}$, allowing to regard either the ion- or electron continuity equation to determine the density evolution. We can, however, not use a corresponding relation for $n_{i m p}$. For cold impurities the drift velocity is given by the $E \times B$ and the ion polarisation drift:

$$
d_{t} n_{\text {imp }}=\frac{M}{Z \widehat{\varepsilon}} \nabla_{\perp} \cdot\left(n_{\text {imp }} d_{t} \nabla_{\perp} \phi\right)-n_{\text {imp }} \mathcal{K}(\phi)-\nabla_{\|}\left(n_{\text {imp }} u\right)-\mu_{\text {imp }} \nabla_{\perp}^{2} n_{\text {imp }}
$$

Here we have introduced the relative mass of the impurities $M=M_{i m p} / M_{i}$ and $Z$ indicates the charge state of the impurity ions. The fluctuating quantities $\phi$ and $u$ are input from the dynamical evolution of the turbulence and the total time derivative $d_{t}$ includes advection with the compressible $E \times B$ velocity.

The dynamical equation for the impurity density thus differs from the dynamical equation for the density fluctuations Eq. (1b). Typical simplifications originating in the distinction between a 
background and fluctuations are not possible to introduce for the impurity species. Finite inertia effects of the impurity ions enter through the ion-polarisation drift and finally all convection terms need to be preserved, while for example parallel convection is neglected in the dynamical equation for the density fluctuations. Note that the equation for the impurity species do not reflect the properties of the equilibrium as given by Eqs. (3) and (4) as the impurities are not assumed to contribute significantly to the establishment of quasi-neutrality.

Simulations were performed on a grid with $128 \times 512 \times 32$ points and dimensions $64 \times 256 \times 2 \pi$ in $x, y, s$. Standard parameters for the runs were $\widehat{\mu}=5, q=3$, magnetic shear $\widehat{s}=1$ (appearing only in the geometrical setup of the simulations [7] ), and $\omega_{B}=0.05$, with the viscosities set to $\mu_{\omega}=\mu_{n}=0.025$, corresponding to typical edge parameters of large fusion devices.

In the saturated turbulent state the equilibrium density gradient is weakly flattened. Strong deviations from the initially specified density gradient are, however, prevented by a feed-back mechanism using two damping layers at the inner and outer radial boundary. This forces the flux-surface averaged density to stay close to its initially specified equilibrium profile.

To investigate impurity diffusion in fully developed, quasi-stationary turbulence we numerically solve Eqs. (1) and let initial perturbations develop until a state of saturated, quasistationary turbulence is reached. The impurities are then released into the turbulence and convected around by the turbulence according to the evolving turbulent velocity field. The initial impurity density $n_{\text {imp }}$ is chosen as a radially localized Gaussian added to a constant impurity background density. For some runs the impurity density was additionally localized along the magnetic field lines, that is in coordinate $s$, to investigate the effect of parallel convective transport. Here we choose to investigate the behavior of massless impurities. The coupled system of bulk plasma turbulence and impurities is evolved until significant mixing of the impurities has been achieved and initial transient effects have decayed. For each parameter several runs are performed to increase the statistical significance of the results.

From the transport of passive fields in compressible fluid turbulence it is well known that the passive quantity reveals a much flatter fluctuation spectrum than the turbulent energy spectrum, moreover the passive scalar tends to accumulate in the contracting regions of the turbulent flow field [2]. Thus, the impurity density after some time not only exhibits strong gradients, but locally the initial value of the impurity density can be exceeded. To be able to handle these effects within the given limited resolution the diffusive term in Eq. (5) was chosen as $\mu_{i m p}=5 \mu_{n}$

A prominent feature of the impurity behavior is the weak parallel convective transport com- 
pared to the radial turbulent transport. The reason is that the impurities are convected in the parallel direction by the fluctuating parallel ion speed $u$ which is small $u \approx 0.01$ compared to a radial velocity that is of order one. This is clearly observed in Fig. 1 which shows the impurity density projected onto a geometrically poloidal cut. The projection roughly translates the variation in $s$ to a poloidal variation. $s=0$ corresponds to the outboard midplane, $s=\pi / 2$ to the upper, $s=-\pi / 2$ to the lower side and finally $s= \pm \pi$ corresponds to the high field side. The radial extend of the simulation domain has been stretched to allow better visualization. The initial impurity density is localized at the outboard midplane, corresponding to a parallel localization in the flux tube geometry. No significant parallel flow of the impurity density is observed, while significant radial mixing occurs. Parallel compressional effects are however visible and arrange for finite passive density gradients at the high field side. Moreover an inward pinch effect is clearly observed at the outboard midplane.

By starting from an initial impurity distribution that is homogeneous along $s$, this pinching velocity is seen to lead to a shift towards the torus axis of the impurity density compared to the initial condition (Figure 2).

For a more quantitative description of this behavior, the flux $\Gamma$ of the impurity ion species can in lowest order be expressed by a diffusion coefficient $D$ and a convective velocity $V$, which is associated to a pinch effect:

$$
\Gamma_{y}(s)=-D(s) \partial_{x}<n>_{y}+V(s)<n>_{y} .
$$

The turbulence is radially homogeneous and so there is no radial dependence of $D$ and $V$. Averages are taken along the periodic $y$ direction. We obtain these values for each value of $s$ along the magnetic field lines and thus at different poloidal positions. From a scatter plot of $\Gamma(r) /<n>y$ versus $\partial_{x}<\ln n>_{y}$ the parameters $D(s)$ and $V(s)$ can be obtained. These are standard parameters used in modeling and in evaluation of transport experiments. The fitting procedure is carried out using a standard nonlinear least-squares Marquardt-Levenberg algorithm [12] as implemented in the GNUPLOT software. Figure 3 shows such a typical scatter plot with a fitted linear relation between the two quantities indicating that while there are significant deviations from a linear relationship, the decomposition of the transport into $D$ and $V$ has some merit. The comparison of the evolution of the impurity density profile with the analytical evolution of the profile using the values for $D$ and $V$, obtained from an analysis as shown in Figure 3 is depicted in Figure 4

The poloidal dependence of diffusion and effective convection is rather strong and depicted in 
Fig. 5. The effective convective velocity $V(s)$ changes sign and is at the high field side directed outwards. This pinching velocity is due to normal curvature and can be consistently explained in the framework of Turbulent EquiPartition (TEP) [1, 13]. In the absence of parallel convection, finite mass effects and diffusion Eq. (5) has the following approximate Lagrangian invariant

$$
L(s)=\ln n_{i m p}+\omega_{B} \cos (s) x-\omega_{B} \sin (s) y
$$

Its spatial homogenization on each drift plane $\langle L(s)\rangle_{y}=\operatorname{const}(s)$ by the turbulence indicates that at the outboard midplane $(s=0)$ the impurites are effectively convected radially inward leading to a radially gradient $\left(<\ln n_{i m p}>_{y} \propto\right.$ const. $\left.-\omega_{B} x\right)$ while at the high field side they are effectively convected radially outward $\left(<\ln n_{i m p}>_{y} \propto\right.$ const. $\left.+\omega_{B} x\right)$. One should note that this effective inward or outward convection is not found as an average $E \times B$ velocity, but is mitigated by the effect of homogenization of $L$ under the action of the turbulence. The strength of the "pinch" effect is consequently proportional to the mixing properties of the turbulence and thus to lowest order has an additional variation that scales with the measured turbulent diffusion, so that $V(s) \propto-\cos (s) D(s)$. The slight ballooning in the turbulence level thus causes the inward flow on the outboard midplane to be stronger than the effective outflow on the high-field side. Averaged over a flux surface and assuming poloidally constant impurity density a net impurity inflow results. The net pinch is directly proportional to the diffusion coefficient $D$ in agreement with experimental observations [14]. Translated to dimensional values for typical large tokamak edge parameters we obtain $D(s) \propto 1.5-2.0 \mathrm{~m}^{2} / \mathrm{s}$ and $V(s) \propto+60--80 \mathrm{~m} / \mathrm{s}$ and an flux-surface averaged inward convection velocity of $\langle V\rangle=-0.4 \mathrm{~m} / \mathrm{s}$. Locally at the outboard midplane values of $V(s=0) / D(s=0) \sim-40 \mathrm{~m}^{-1}$ are found, in rough agreement with experimental values [3].

Furthermore we observe a slight shift of the peak diffusion coefficient in the diamagnetic direction. This effect is likely due to the spreading of the turbulence [15] in that direction leading to enhanced turbulence levels slightly upward from the outboard midplane and consequently to an up-down asymmetry.

The strong peaking of the impurity density, the poloidal dependence of the transport coefficients and the slow parallel diffusion of the impurities thus make it necessary to apply at least two dimensional modeling. Both a poloidally varying diffusion coefficient and effective convection velocity should be used in transport codes to describe impurity density evolution. The observed impurity pinch in the edge plasma region can be explained by turbulent equipartition without invoking arguments from neoclassical transport theory. 
This work was supported by the Danish Center for Scientific Computing through grants no. CPU-1101-08 and CPU-1002-17. Discussions with K.D. Zastrow and X. Garbet during a stay of the author at JET are gratefully acknowledged.

[1] J. Nycander and V. V. Yan'kov, Phys. Plasmas 2, 2874 (1995).

[2] G. Falkovich, K. Gawdzki, and M. Vergassola, Rev. Mod. Phys. 73, 913 (2001).

[3] R. Dux, Fusion Science and Technology 44, 708 (2003).

[4] M. E. Puiatti et al., Plasma Phys. Contr. Fusion 45, 2011 (2003).

[5] K. D. Zastrow, Nucl. Fusion 39, 1891 (1999).

[6] P. H. Rutherford, Phys. Fluids 17, 1782 (1974).

[7] B. D. Scott, Plasma Phys. Control. Fusion 39, 471 (1997).

[8] B. D. Scott, Plasma Phys. Control. Fusion 39, 1635 (1997).

[9] V. Naulin, Phys. Plasmas 10, 4016 (2003).

[10] B. D. Scott, Phys. Plasmas 8, 447 (2001).

[11] S. I. Braginskii, in Reviews of Plasma Physics, edited by M. A. Leontovich (Consultants Bureau, New York, 1965).

[12] D. Marquardt, Journal of the Society for Industrial and Applied Mathematics 11, 431 (1963).

[13] V. Naulin, J. Nycander, and J. Juul Rasmussen, Phys. Rev. Lett. 81, 4148 (1998).

[14] M. E. Perry et al., Nucl. Fusion 31, 1859 (1991).

[15] Z. Lin and T. S. Hahm, Phys. Plasmas 11, 1099 (2004). 

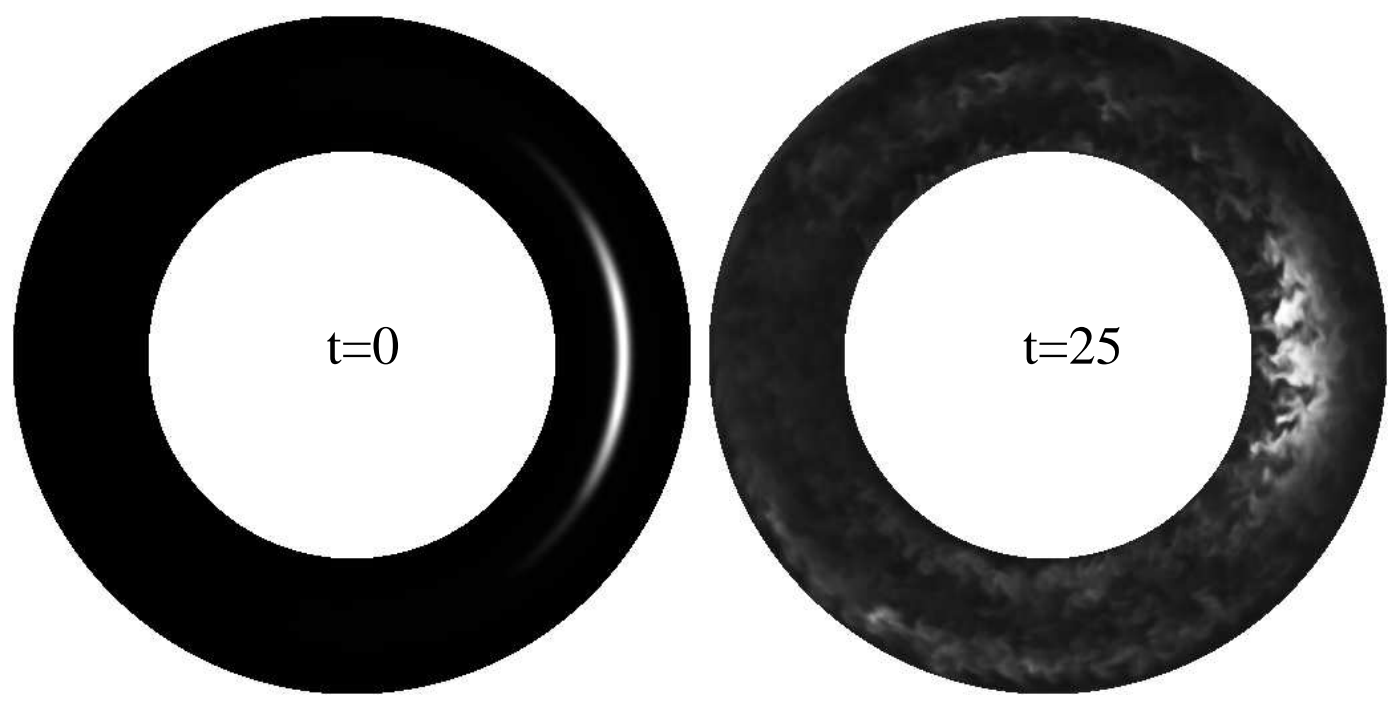

FIG. 1: Impurity distribution projected onto a poloidal cross-section (note: radial dimension not to scale), white: high impurity density and dark: low impurity density. Left: initial distribution, localized along the magnetic field on the outboard midplane (at $s=0$. Right: after 25 time units corresponding to about $100 \mu \mathrm{s}$. Parallel transport is slow compared to radial transport. The inward pinch effect is clearly visible. 

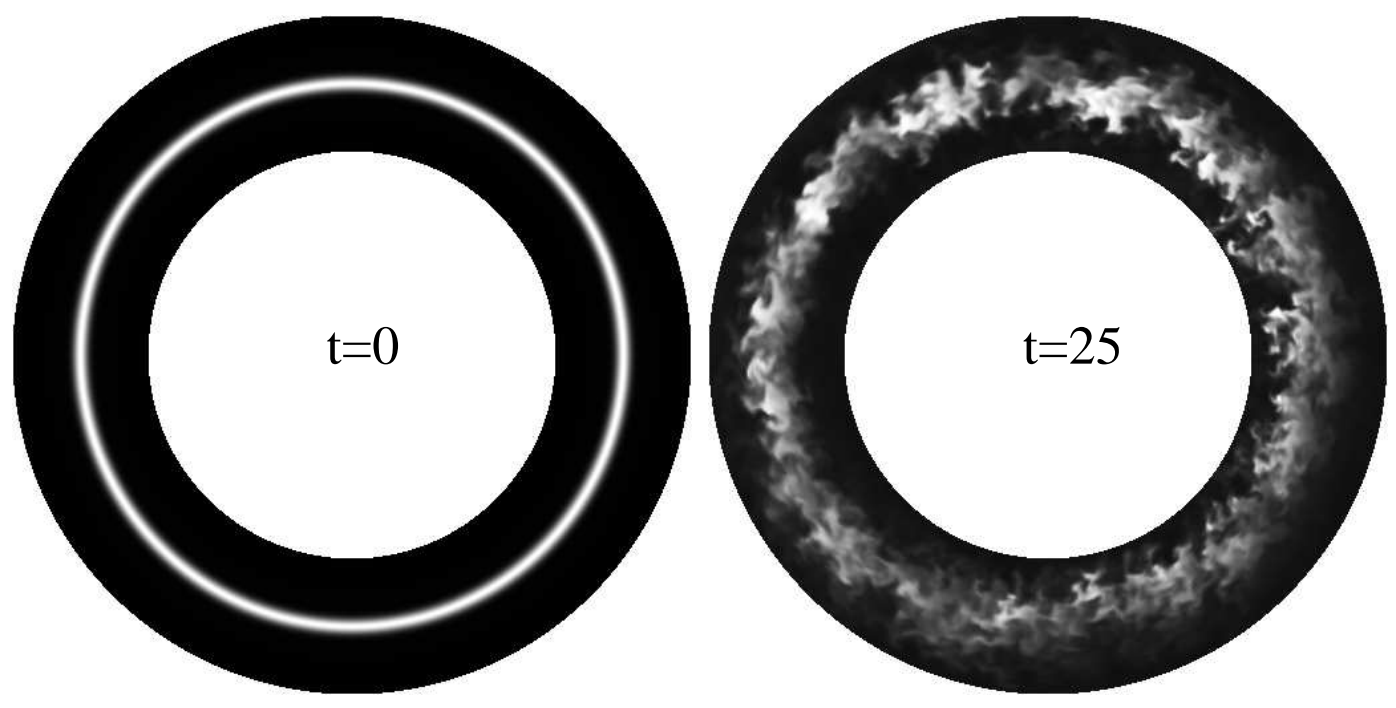

FIG. 2: Impurity distribution projected onto a poloidal cross-section (note: radial dimension not to scale). Left: initial distribution. Right: after 25 time units. The inward pinch effect on the outboard midplane and outward convective transport on the high field side (inboard midplane) is obvious. 


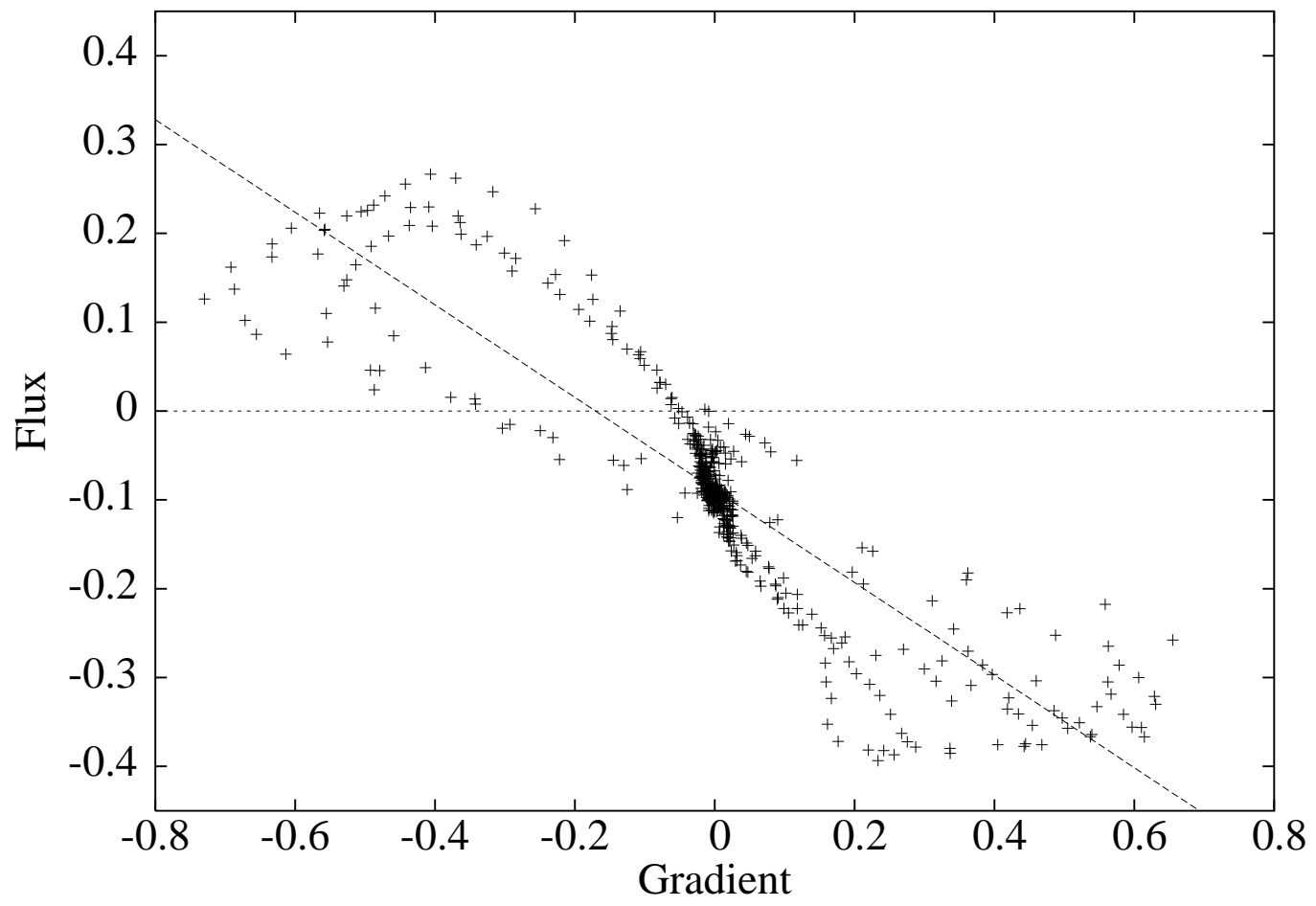

FIG. 3: Typical scatter plot (at high field side) of the flux versus gradient with linear fit. 


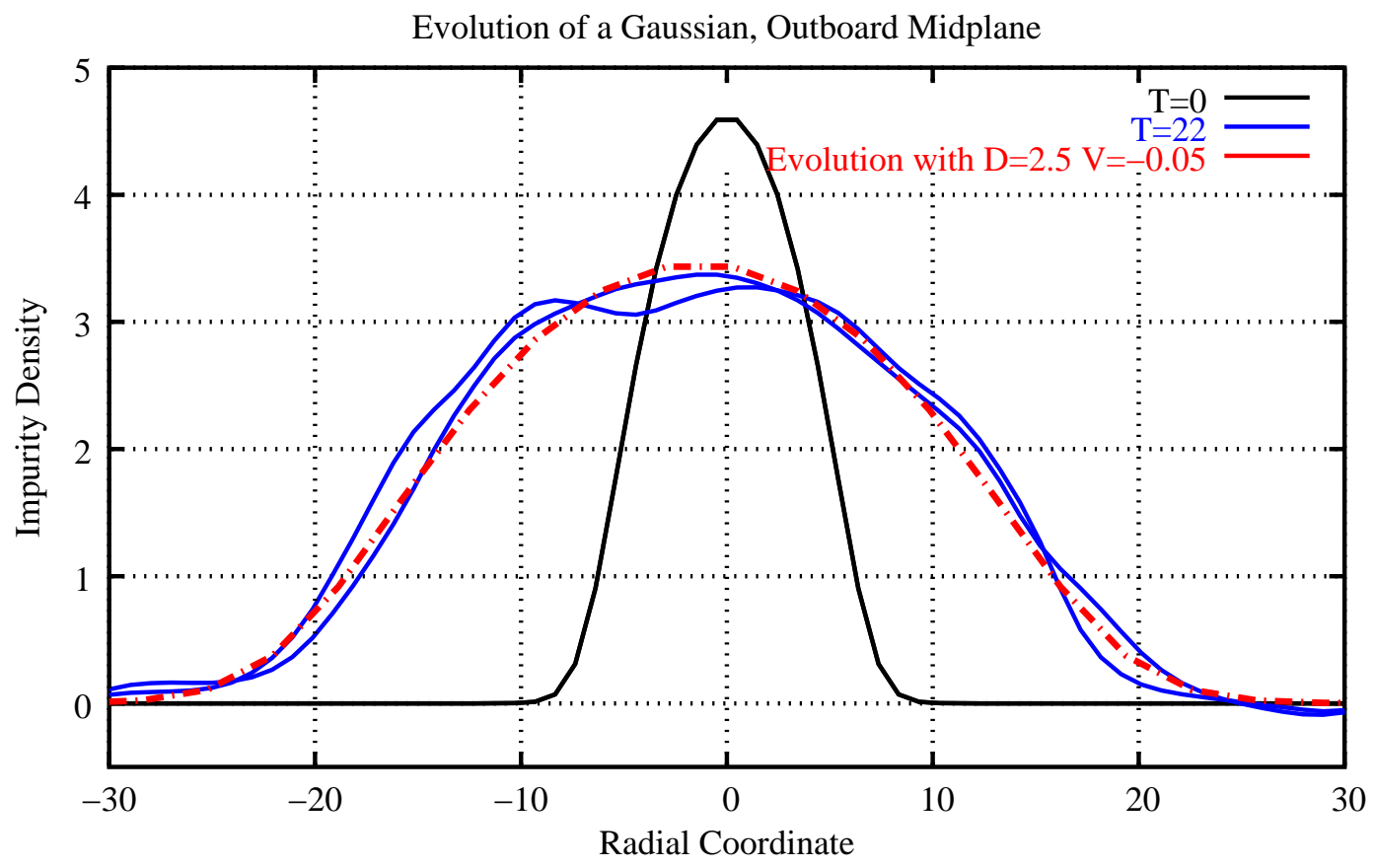

FIG. 4: Impurity density averaged along $y$ at the outboard midplane and compared to the expected evolution of a Gaussian from the fitted coefficients $D$ and $V$. 

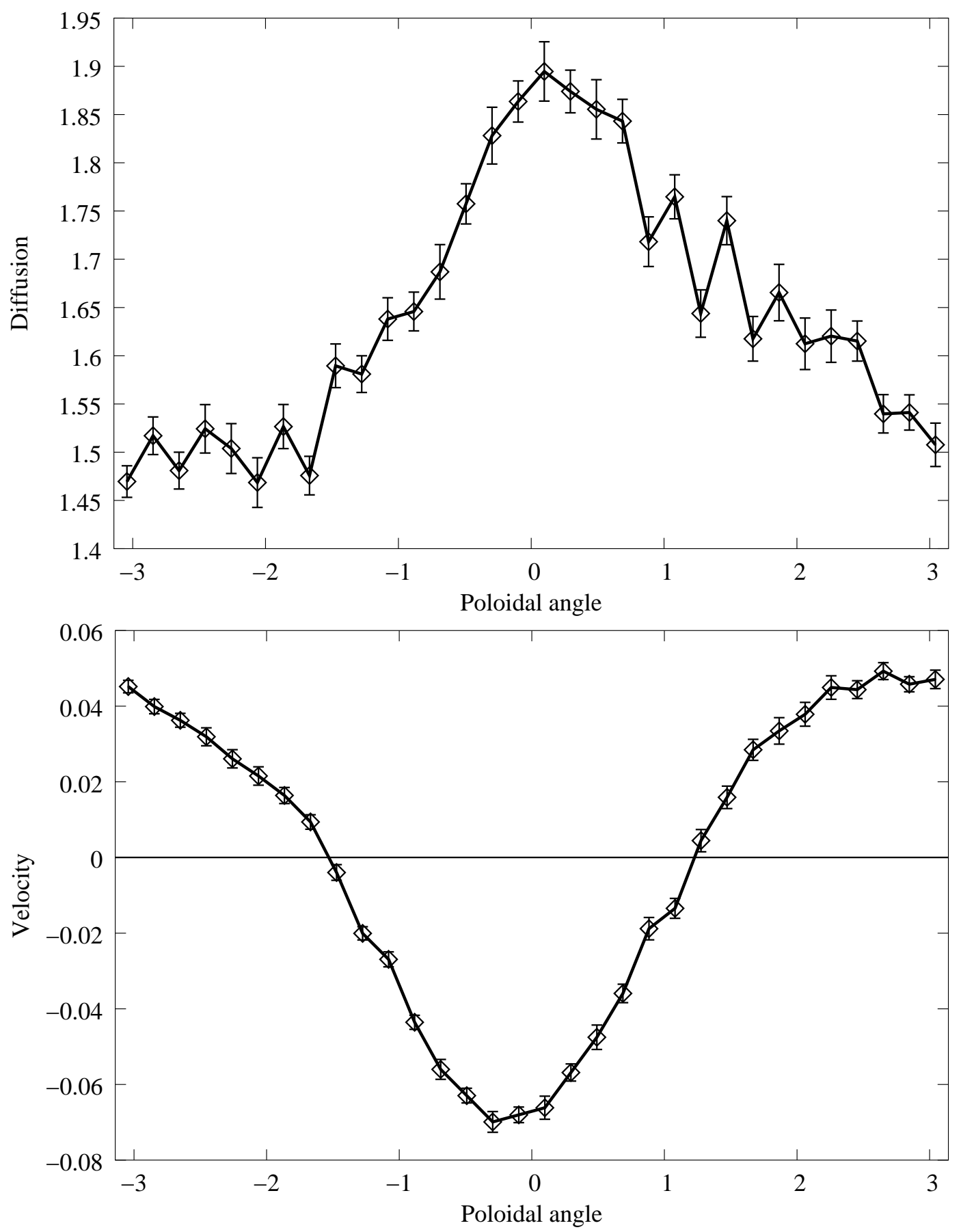

FIG. 5: Impurity diffusion $D$ (top) and pinch velocity $V$ over poloidal position angle with indicated errorbars. The observed asymmetry is due to turbulence spreading in the diamagnetic direction. 